\title{
Biofilm formation at the air-liquid interface by the Pseudomonas fluorescens SBW25 wrinkly spreader requires an acetylated form of cellulose
}

\author{
Andrew J. Spiers, ${ }^{1 *}$ John Bohannon, ${ }^{1}$ \\ Stefanie M. Gehrig' and Paul B. Rainey ${ }^{1,2}$ \\ ${ }^{1}$ Department of Plant Sciences, University of Oxford, \\ South Parks Road, Oxford OX1 3RB, UK. \\ ${ }^{2}$ School of Biological Sciences, University of Auckland, \\ Private Bag 92019, Auckland, New Zealand.
}

\section{Summary}

The wrinkly spreader (WS) genotype of Pseudomonas fluorescens SBW25 colonizes the air-liquid interface of spatially structured microcosms resulting in formation of a thick biofilm. Its ability to colonize this niche is largely due to overproduction of a cellulosic polymer, the product of the wss operon. Chemical analysis of the biofilm matrix shows that the cellulosic polymer is partially acetylated cellulose, which is consistent with predictions of gene function based on in silico analysis of wss. Both polar and non-polar mutations in the sixth gene of the wss operon (wssF) or adjacent downstream genes (wssGHIJ) generated mutants that overproduce non-acetylated cellulose, thus implicating WssFGHIJ in acetylation of cellulose. WssGHI are homologues of AlgFIJ from P. aeruginosa, which together are necessary and sufficient to acetylate alginate polymer. WssF belongs to a newly established Pfam family and is predicted to provide acyl groups to WssGHI. The role of WssJ is unclear, but its similarity to MinD-like proteins suggests a role in polar localization of the acetylation complex. Fluorescent microscopy of Calcofluor-stained biofilms revealed a matrix structure composed of networks of cellulose fibres, sheets and clumped material. Quantitative analyses of biofilm structure showed that acetylation of cellulose is important for effective colonization of the air-liquid interface: mutants identical to WS, but defective in enzymes required for acetylation produced biofilms with altered physical properties. In addition, mutants producing non-acetylated cellulose were unable to spread rapidly across solid

Accepted 11 June, 2003. *For correspondence. E-mail andrew.spiers@plants.ox.ac.uk; Tel. (+44) (0) 1865 275000; Fax (+44) (0) 1865275074.

(C) 2003 Blackwell Publishing Ltd surfaces. Inclusion in these assays of a WS mutant with a defect in the GGDEF regulator (WspR) confirmed the requirement for this protein in expression of both acetylated cellulose polymer and bacterial attachment. These results suggest a model in which WspR regulation of cellulose expression and attachment plays a role in the co-ordination of surface colonization.

Introduction

Colonization of a new niche by bacteria, irrespective of whether that niche is a mammalian or plant host, soil or water, usually involves growth at a surface and subsequent formation of a biofilm. There are many consequences (both positive and negative) of growth at a surface: attached cells are less likely to be displaced by physical processes, they are better able to withstand competition from newly invading organisms, they are more resistant to predation, more resilient to the challenges posed by host immune responses, and during initial stages of colonization individual cells may benefit from nutritional opportunities that result from concerted activity of the entire colony (for reviews see Costerton et al., 1995; Roberts, 1996; Davey and O'Toole, 2000; Lappin-Scott and Bass, 2001; Sutherland, 2001; Wilson, 2001; Stephens, 2002). At the same time, attached cells are often unable to escape degrading environments, and unless dispersal is possible, they are likely to fall victim to their initial success (Hamilton and May, 1977).

Attachment is the first stage in the formation of a biofilm and subsequent growth often occurs concomitantly with the production of an extensive network of exocellular polymers (often exopolysaccharides) that facilitate firm adherence of bacteria to daughter cells and to the surface (Kuchma and O'Toole, 2000; Sutherland, 2001; Wilson, 2001). Biofilm architectures are highly variable, ranging from open structures containing channels and columns of bacteria, to structures with no obvious pores and densely packed regions of cells (Lawrence et al., 1991; Watnick and Kolter, 1999, 2000; Wimpenny et al., 2000). The close physical association of cells within these biofilms results in a structure with significant phys- 
ical properties and changes in bacterial physiology compared with free-living cells (Costerton et al., 1995; Kuchma and O'Toole, 2000; Wimpenny et al., 2000; Sutherland, 2001).

To date most attention has focused on biofilms that arise from the colonization of solid-liquid (S-L) interfaces (i.e. submerged biofilms), but several other kinds of surface and interface also provide ecological opportunity for bacteria. The most common of these is the interface between air and liquid (A-L interface), which when colonized, provides bacteria with access to both the gaseous (e.g. oxygen) and liquid (e.g. nutrient) phases. Given the potential benefits to aqueous-dwelling aerobic bacteria, it is of interest that colonization of the $A-L$ interface has been rarely studied and that there are few bacteria reported to be capable of colonizing this niche.

Most bacteria when propagated in static liquid culture grow within the broth phase or quickly sediment to the bottom. However, when propagated for 20 generations or more in a spatially structured environment (unshaken broth culture), mutants with an enhanced ability to colonize the $A-L$ interface frequently arise (Rainey and Travisano, 1998; Römling and Rohde, 1999; Solano et al., 2002). The biofilms generated by these mutants firmly adhere to the microcosm walls and their hydrophobic nature (rather than buoyancy) ensures that they remain at the A-L interface. In Salmonella typhimurium, S. enteritidis and Pseudomonas fluorescens the A-L interface colonizing mutants all generate distinctive dry, wrinkled colonies on agar plates (Römling and Rohde, 1999; Zogaj et al., 2001; Solano et al., 2002; Spiers et al., 2002). In S. typhimurium and $S$. enteritidis these morphs are referred to as rdar (multicellular and aggregative behaviour) types (Römling and Rohde, 1999; Zogaj et al., 2001; Solano et al., 2002); the diverse range of similarly shaped colonies that evolve from the ancestral smooth (SM) genotype of $P$. fluorescens SBW25 when it is propagated in a spatially structured environment are known as wrinkly spreaders (WS) (Rainey and Travisano, 1998). In S. typhimurium, $S$. enteritidis, and $P$. fluorescens, the A-L interface colonizing mutants show enhanced activation of traits associated with attachment and surface colonization; all overproduce a cellulose polymer and in all instances this polymer has been shown to be a primary cause of $A-L$ interface colonization (Solano et al., 2002; Spiers et al., 2002).

The cellulose-like polymer (CLP) produced by $P$. fluorescens SBW25 is encoded by a 10-gene operon termed wss (Fig. 1) (Spiers et al., 2002). On the basis of amino acid homology with Gluconacetobacter xylinus (formerly Acetobacter) bacterial cellulose synthase (bcs) proteins, WssB, WssC and WssE have been identified as the cellulose synthase subunits (for a recent review of bcs see Römling, 2002). WssD is an associated cellu- lase required for normal cellulose synthesis in G. xylinus. The function of WssF is unknown, but it is unlikely to be essential for cellulose expression given that it is only rarely found associated with cellulose biosynthetic operons (Römling, 2002). WssG, WssH and Wssl are so far reported to be unique to the wss operon and show homology to the $P$. aeruginosa proteins AlgF, Algl and AlgJ (38, 61 and 48\% similarity, respectively), which together are responsible for O-acetylation of alginate (Franklin and Ohman, 1996), suggesting that the wss operon encodes a modified (acetylated) form of cellulose (ACP). Finally, WssA and WssJ both show strong homology with MinD, a highly conserved prokaryote protein, which in E. coli is involved in cell-cycle control and positioning of the division septum (Sullivan and Maddock, 2000). It is likely that WssA and WssJ act to position the cellulose synthase complex at the $P$. fluorescens cell poles (Spiers et al., 2002). In P. fluorescens SBW25 the wss cellulose synthase complex is regulated by a chemosensory pathway termed Wsp (Spiers et al., 2002; E. Bantinaki, A. J. Spiers and P. B. Rainey, unpubl. obs.). The seventh gene of this cluster, $w s p R$, encodes a CheY-GGDEF like protein that activates expression of cellulose at a post-transcriptional level (P. Goymer, A. J. Spiers and P. B. Rainey, unpubl. obs.).

Previous analysis of $P$. fluorescens SBW25 WS defective (WS ${ }^{-}$) mutants revealed two distinct phenotypes associated with mini-Tn5-km transposon insertions within the wss locus. Strains with transposon insertions in the first five genes of the wss operon are cellulose negative, whereas those with insertions in the sixth gene of the operon (wspF) or beyond (WS-18 in Fig. 1) continue to overproduce the polymer (as determined by a simple Congo Red-based plate assay). wss$A B C D E$ mutants are completely defective in A-L-biofilm formation, whereas wssFGHIJ mutants (WS-6, WS-9 and WS-18) produce a weak biofilm at the A-L interface (Spiers et al., 2002) (Fig. 2). In addition, wssFGHIJ mutants lose their ability to spread rapidly across solid surfaces - a characteristic ability of WS (see Fig. 2D

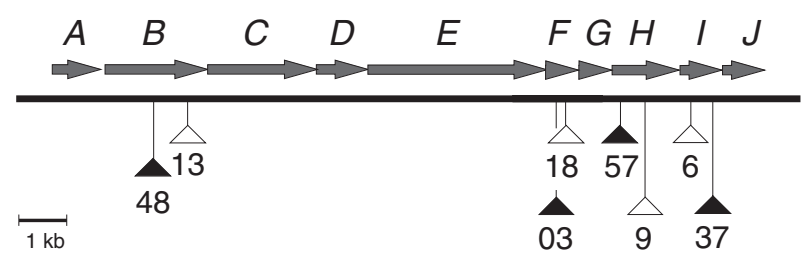

Fig. 1. The wss operon from Pseudomonas fluorescens SBW25 is composed of 10 genes, wssA-J. WssBCDE are cellulose synthase subunits, WssFGHI are predicted to be involved in cellulose acetylation, and WssA and $\mathrm{J}$ are predicted to play a role in the correct cellular localization of the synthase/acetylation complex. The locations of the WS-6, 9, 13 and 18 mini-Tn5 insertions (Spiers et al., 2002), and the LSWS-37, 48, 57 and P-03 ISphoA/hah insertions are indicated. 


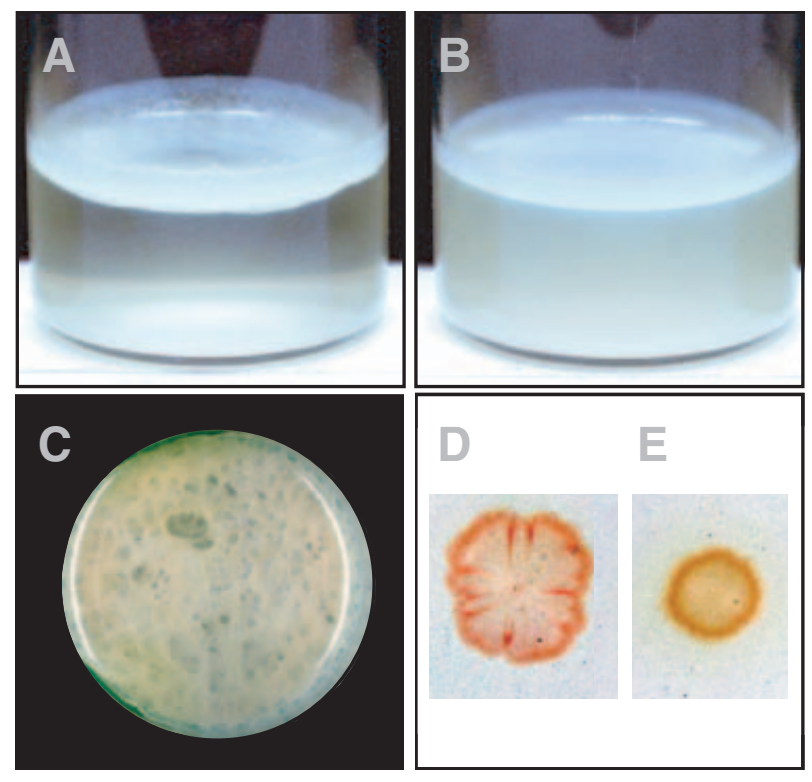

Fig. 2. WS and WS-18 A-L biofilms and colony morphologies differ. A. WS biofilm viewed from the side compared with the substantially weaker WS-18 (WS mini-Tn5::wspF) biofilm, B.

C. The WS biofilm viewed from above reveals a clear physical structure.

D. WS colonies show characteristic wrinkled-spreading morphology and stain red when grown on LB containing Congo Red.

E. Colony morphology, spreading behaviour and binding of Congo Red by WS-18.

and E). This observation suggested that acetylation of the core cellulose polymer may play a biologically significant role in A-L biofilm formation and in the rapid surface-spreading of $P$. fluorescens SBW25 WS colonies. Here we describe the results of chemical analyses of the cellulosic product of the wss operon and provide evidence that the operon also contains genes responsible for acetylation of the cellulose polymer. We also show that acetylation of cellulose is a critical factor in the formation of the WS A-L biofilm and in rapid colony spreading.

\section{Results}

Comparison of polar and non-polar insertion mutants in the wsSFGHIJ region

Initial analysis of the mini-Tn5 mutants WS-6, WS-9 and WS-18 indicated that these strains were phenotypically indistinguishable from one another (Spiers et al., 2002), and, as a result, WS-18 was chosen as a representative mutant for further analysis. Subsequently, as part of a larger effort to screen for genes involved in the WS phenotype we isolated three ISphoA/hah insertion mutants in wssF, wssH and wssl (Fig. 1). Like mini-Tn5, the ISphoA/ hah transposon disrupts gene function at the point of insertion and has a polar effect on downstream gene expression. However, non-polar mutants can be obtained by Cre-deletion of ISphoA/hah which leaves a 63 amino acid fragment is left at the site of insertion (Bailey and Manoil, 2002).

P-03 (ISphoA/hah::wssF), LSWS-37 (ISphoA/ hah::wsSI) and LSWS-57 (ISphoA/hah::wssH) produce SM-like colonies phenotypically indistinguishable from the mini-Tn5 mutants WS-6, WS-9 and WS-18. All three expressed CLP as assessed using Congo Red agar plates and produced weak $A-L$ interface biofilms in KB microcosms. The Cre-deletions of these three mutants ( $P$ 03 $\Delta$, LSWS-37 $\Delta$ and LSWS-57 $\Delta$ ) similarly produced SMlike colonies, expressed CLP and formed weak biofilms at the A-L interface (the presence of CLP was confirmed by Calcofluor-staining of colony material and examination by fluorescent microscopy). In contrast, the Cre-deletion of LSWS-48 (WS ISphoA/hah::wssB) produced SM-like colonies on agar plates containing Congo Red, did not express CLP or produce a biofilm, indicating that the 63 amino acid insertion left at the site of the Cre-deletion was sufficient to disrupt gene function in the WS.

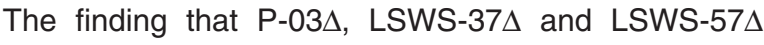
show the same phenotype as the mini-Tn5 mutants WS-6, WS-9 and WS-18 allow the following conclusions to be drawn: (i) wssFGHIJ mutants can not be subdivided on the basis of phenotypic differences; and (ii) additional polar effects on downstream gene expression in wssF, wssH or wssl mutants do not alter the mutant phenotype.

\section{A quantitative assay for CLP}

To test the hypothesis that differences among wsSABCDE and wSSFGHIJ mutants were attributable to the activity of WssFGHI and not simply a reduction in the amount of core cellulosic material produced by the wssFGHI mutants, a quantitative assay for the cellulosic product was developed. This assay was based upon uptake of Congo Red (CR), a dye that binds (1-4)- $\beta$-D-glucopyranosyl units (Weiner et al., 1999). Briefly, cells were incubated in a solution of $\mathrm{CR}$ and the amount of dye removed through binding was determined by measuring the $\mathrm{OD}_{490}$ of the solution after the removal of biofilm material or cells. Initial experiments using WS biofilm material showed that over the range of cellulose concentrations used here the doseresponse was linear. The results presented in Table 1 show that WS and the wsSFGHIJ mutants (WS-6, WS-9 and WS-18) produce approximately twice the amount of CR-binding material compared to both the ancestral broth-colonizing SM genotype and the WS miniTn5::wsp $R$ regulatory mutant (WS-4).

These data demonstrate that differences in colonization of the A-L interface and rapid surface spreading by WS 
Table 1. Congo Red (CR)-binding by P. fluorescens strains.

\begin{tabular}{lll}
\hline Strain & CR-binding $\left(\mathrm{mg} \mathrm{CR} / \mathrm{OD}_{600}\right)$ & Relative CR-binding \\
\hline SM & $0.222 \pm 0.011$ & 1.00 \\
WS & $0.394 \pm 0.012$ & 1.78 \\
WS-4 & $0.219 \pm 0.012$ & 0.99 \\
WS-6 & $0.416 \pm 0.015$ & 1.87 \\
WS-9 & $0.400 \pm 0.017$ & 1.80 \\
WS-13 & $0.272 \pm 0.025$ & 1.23 \\
WS-18 & $0.429 \pm 0.039$ & 1.93 \\
\hline
\end{tabular}

Colonies grown on LB agar plates for $24 \mathrm{~h}$ before assay. Total Congo Red (CR)-binding determined by measuring $\mathrm{mg}$ bound-CR/OD 600 (mean $\pm \mathrm{SE}, n=5$ ). Relative CR-binding is calculated relative to SM.

are attributable to the effects of WssFGHIJ on properties of the cellulosic polymer. They also provide confirmation of the previously described phenotypic equivalence of the wssFGHIJ mutants (WS-6, WS-9 and WS-18) (Spiers et al., 2002). This led to us to base all subsequent work on a single representative mutant, WS-18 (wssF::mini-Tn5-km).

An unexpected finding was the greater than anticipated amount of CR-binding material produced by the WS miniTn5:: wssB mutant (WS-13) 1.25-fold greater than the ancestral (control SM strain). This suggested that WS-13 produced cellulosic polymer, even though sequencing confirmed the presence of the transposon within the second gene of the operon (Fig. 1). Congo Red is known to bind some non-cellulosic materials in addition to cellulose, such as certain proteinacious fimbriae (Collinson et al., 1993; Hammar et al., 1995; Solano et al., 2002). We therefore repeated the $\mathrm{CR}$ assay on material pretreated with cellulase to determine whether a non-cellulose CRbinding component was present. Both WS and WS-18 produced a significant non-cellulosic CR-binding component ( $30 \%$ of the total CR-binding activity), which was not detected in the SM genotype or in the WS mini-Tn5::wspR mutant (WS-4). In contrast, $100 \%$ of the CR-binding material produced by WS-13 was found to be non-cellulosic and this was confirmed by Calcofluor staining and fluorescent microscopy (Calcofluor is highly specific for cellulose, Weiner et al., 1999). These results indicate that WS, WS13 and WS-18 express an additional non-cellulosic CRbinding component not expressed by other strains.
Moreover, as the non-cellulosic CR-binding component was not detected in WS-4, we conclude that expression of both the cellulosic and non-cellulosic CR-binding components are dependent upon WspR.

Given our suspicion that the non-cellulosic CR-binding component is a proteinacious fimbrial-like structure, we examined cells of SM, WS and the WS mini-Tn5::wsSB mutant (WS-13) by transmission electron microscopy (TEM). Analysis of many cells of each genotype revealed clear evidence of a fimbrial-like extension in WS that was absent from ancestral SM, but present in WS-13 (Fig. 3). Detection of this component in both WS and WS-13 suggests that this fimbrial-like extensin is the non-cellulosic CR-binding component.

Chemical and linkage analysis of the WS and WS-18 A-L biofilm matrices

In order to confirm the basic chemical nature of the cellulosic polymer produced by WS and to test the hypothesis that the WS mini-Tn5:: wSSF mutant (WS-18) produces a non-acetylated form of cellulose, we undertook a chemical composition and linkage analysis of the matrix material isolated from WS and WS-18 biofilms. Initial composition analysis of WS biofilm material identified significant amounts of glucose (Glu), rhamnose (Rha) and 3-deoxyD-amino-2-octulosonic acid (Kdo) (plus trace amounts of other hexoses and fatty acids). The presence of Rha and Kdo was indicative of lipopolysaccharide (LPS) contamination of the matrix material; Kdo is an absolutely conserved component of the core LPS molecule, whereas the Pseudomonas A-band O-polysaccharide component is composed of a Rha polymer of 50-60 subunits (Rocchetta et al., 1999). In order to confirm this possibility, we determined the chemical composition of carbohydrate material from WS, WS-6 (WS mini-Tn5::wssl), WS-18 biofilms and an equivalent pelleted mass of SM culture. In all four cases, we observed similar levels of Rha $(12-17 \%$ of sample) and Kdo (3-7\%). The fact that Rha and Kdo could be identified from the SM extract indicates that these components are not part of the WS matrix material. As Rha and Kdo were also found in the WS- 6 and WS-18 extracts, they do not provide an explanation for the differ-

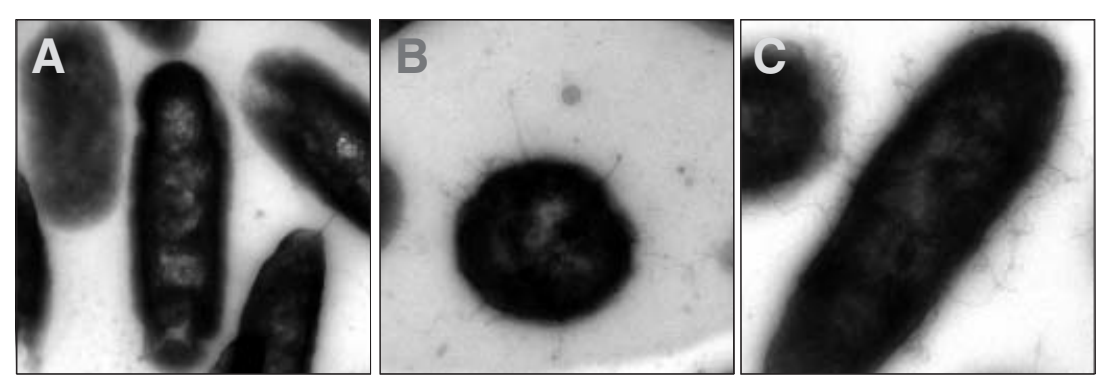

Fig. 3. Transmission electron microscopy (TEM) of osmonium stained SM, WS and WS13 (WS mini-Tn5::wssB) cells. Filamentous structures were consistently observed extending from WS and WS-13 cells, but were absent from SM. TEM micrographs of A, SM cells; B, WS; and C, WS-13. 
ence seen between the biofilms produced by the WS and acetylation-deficient mutants.

By modifying our matrix isolation procedure by extending enzymatic digestion times and including an extensive series of washes before carbohydrate analysis, we substantially improved the isolation of CLP to the degree that the WS and WS-18 samples contained $77.4 \%$ Glu $22.6 \%$ Rha and $69.9 \%$ Glu $-30.1 \%$ Rha respectively. The lack of detectable amounts $(<01 . \%)$ of Kdo and other hexose residues indicated that these samples lacked core LPS but still retained significant amounts of A-band LPS. Significantly, these samples did not contain detectable levels of any other carbohydrate residue that may have been present in the core LPS molecule (the core LPS composition of $P$. fluorescens SBW25 has not been reported, but is known to be highly varied amongst other strains of this bacterium; Naberezhnykh et al., 1987; Knirel et al., 1996; Shashkov et al., 1998; Veremeichenko, 1998; Veremeichenko and Zdorovenko, 1994; 1996; Zdorovenko et al., 1999) which might have confounded our interpretation of the composition of the matrix material itself.

Linkage analysis of derivatized samples of WS matrix material by GC-MS identified a large peak corresponding to 4-Glu, and minor, but significant peaks corresponding to 2,4-Glu, 3,4-Glu and 4,6-Glu (Fig. 4A). The presence of such large amounts of derivatized 4-Glu is consistent with the sample having contained cellulose, a $\beta(1-4)$ - linked glucose polymer. In comparison, an analysis of matrix material extracted from WS-18 biofilms identified significant amounts of 4-Glu but no detectable amounts of 2,4-Glu, 3,4-Glu or 4,6-Glu (<0.1\%) (Fig. 4B). Given that WS and WS-18 differ only in the inactivation of putative cellulose acetylation genes, it is possible to hypothesize that in the WS, the product of these genes acetylates glucose residues at the 2, 3 and 6 Carbon positions in the cellulose polymer. This hypothesis is supported by $\left[{ }^{1} \mathrm{H}\right]-$ NMR analysis which confirmed the presence of acetylated hexose residues in the WS extract (Fig. 4C). Given that this sample contained $77.4 \%$ Glu with no other detectable hexose residues present (Rha is not a hexose), the $\left[{ }^{1} \mathrm{H}\right]-$ NMR signal must relate to acetylated glucose residues.

The relative amounts of 2,4-Glu, 3,4-Glu and 4,6-Glu present in the derivatized linkage analysis samples can be used to estimate the average level of acetylation of cellulose in the WS sample. These data would suggest that some $14 \%$ of glucose residues in the polymer are modified with one acetyl group at the 2, 4 or 6 Carbon positions.

\section{Effect of acetylation on biofilm structure}

Given that the remarkably different phenotypes of WS and WS mini-Tn5::wssF (WS-18) (Fig. 2) are attributable to lack of cellulose acetylation in the latter, we sought to further characterise the biofilm formed by WS in order to
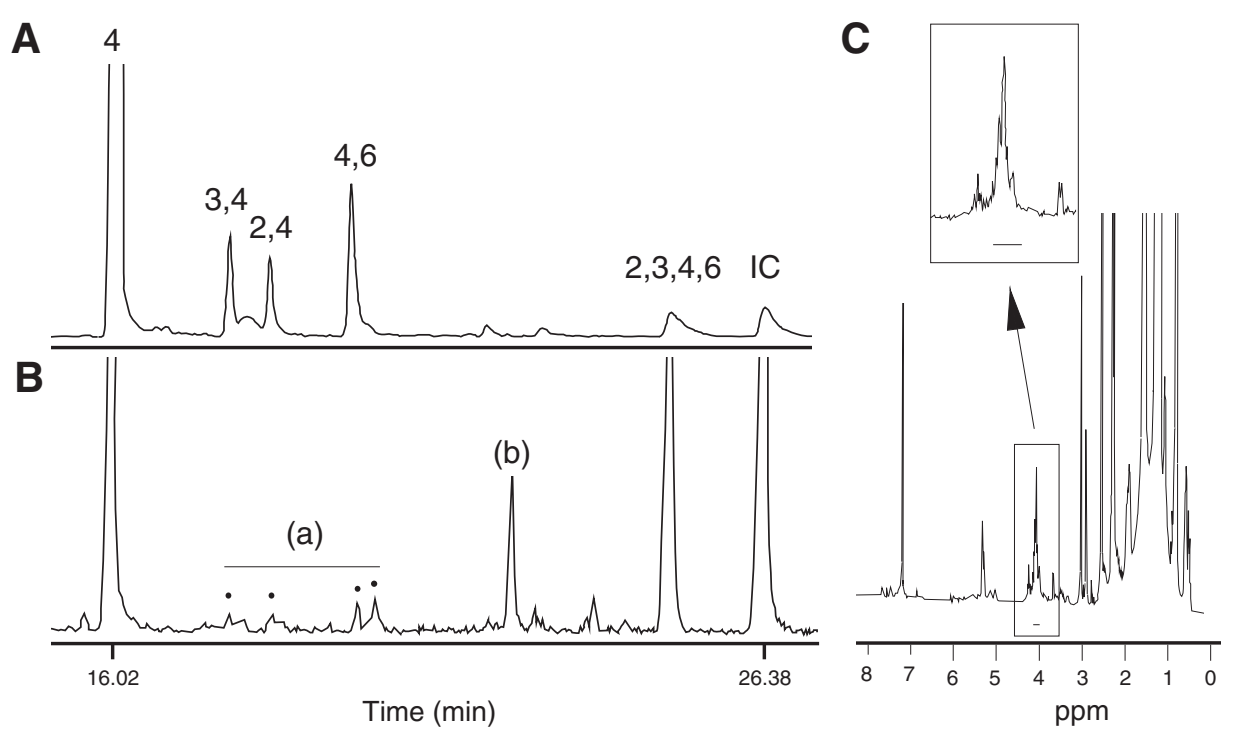

Fig. 4. GS-MS linkage and [1H]-NMR analysis of WS and WS-18 (WS mini-Tn5::wssF) biofilm matrix material. The gas chromatography elution profiles with glucose (Glc) derivatives identified by mass spectrophotometry are indicated.

A. GS-MS profile of processed WS material.

B. GS-MS profile of processed WS-18 material (shown with the peak intensity enhanced by 10x); 4, Glc-4; 2,4, 2,4-Glc; 3,4, 3,4-Glc; 4,6, 4,6Glc; $2,3,4,6,2,3,4,6$-Glc, IC, internal control. The four minor peaks covered by (a) were below the $0.1 \%$ background limit and were not identified as glucose derived residues, and (b) was an unidentified contaminant. 2,3,4,6-Glc is from LPS contamination of the carbohydrate sample.

C. $\left[{ }^{1} \mathrm{H}\right]-$ NMR spectra of WS biofilm material with the peak corresponding to acetylated hexose highlighted and shown enlarged in the insert. The peaks to the right of this were identified as the acetylation peaks of fatty acids present in minor quantities in the sample. 
more precisely determine the consequences of cellulose acetylation on biofilm structure.

The WS A-L biofilm is a visually and physically substantial structure that develops within 2-3 days of inoculation at $28^{\circ} \mathrm{C}$ in static $\mathrm{KB}$ broth-containing microcosms. Within $12 \mathrm{~h}$ of inoculation, the biofilm completely covers the A-L interface $\left(3.8 \mathrm{~cm}^{2}\right)$ and by the fifth day of incubation mature biofilms have a depth of $1-1.5 \mathrm{~mm}$. Mature biofilms have an average wet-weight ( \pm SE) of $0.432 \pm 0.035$

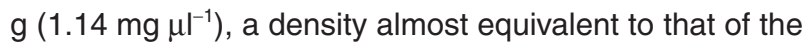
culture media $\left(\sim 1.02 \mathrm{~g} \mathrm{ml}^{-1}\right)$, and an average freeze-dried weight $( \pm \mathrm{SE})$ of $0.014 \pm 0.004 \mathrm{~g}\left(0.036 \mathrm{mg} \mathrm{m}^{-1}\right)$ with a freeze-dried/wet ratio of 0.03 (i.e. $3 \%$ solids). These biofilms contain $9.0 \pm 1.8(\mathrm{SE}) \times 10^{8}$ cells at a density of approximately $2 \times 10^{6}$ cells $\mathrm{ml}^{-1}$.

In order to identify the major limiting factors of $A-L$ biofilm development, nutrient levels and oxygen availability were manipulated. Biofilm development was not limited by nutrient availability because KB medium was able to support the production of a second biofilm by day 11 when the first biofilm was removed on day 6 . Biofilms developed normally in KB lacking glycerol, and development was only retarded once nutrient levels were 0.125 -fold less than those found in KB. In contrast, biofilm development was found to be sensitive to oxygen availability, which was demonstrated by applying a layer of mineral oil to limit oxygen diffusion into the broth. Biofilms developed normally in standard microcosms in which $0.5 \mathrm{ml}$ mineral oil was added to form a layer less than $1 \mathrm{~mm}$ deep. Development was hindered by a $1.5-\mathrm{mm}$ layer $(1 \mathrm{ml})$ of oil, and normal biofilm growth was effectively prevented by a $3 \mathrm{~mm}$ layer $(2 \mathrm{ml}$ ) of oil (there was no toxic effect from the mineral oil, as WS cultures grew normally in shaken microcosms containing this amount of oil).
Fluorescent microscopy in conjunction with the cellulose-specific dye, Calcofluor, was used to investigate the structure of WS A-L biofilms. Biofilms were dominated by the presence of an extensive extracellular network of cellulose fibres (Fig. 5). The network has no scaled structure and fibres ranged in size from less than $0.02 \mu \mathrm{m}$ (less than the width of cells) to over several hundred times the length of cells $(>100 \mu \mathrm{m})$. In places, the fibres aggregated to form larger clumps of material. This clumping was the result of mechanical disturbance, as the more the biofilm samples were manipulated on the microscope slide, the more evident these structures became. In regions, the biofilm resembled cotton wool and large numbers of bacteria were found among the holes and fibres. The bacteria found within the biofilm generally did not stain with Calcofluor, suggesting that most cells were not expressing cellulose at the time of observation. Bacteria were not obviously connected to the cellulose network, although many were confined to large clumps within the network or closely associated with larger fibres.

A-L biofilms produced by WS-6 (WS mini-Tn5::wssl), WS-9 (WS mini-Tn5::wssH) and WS-18 (WS miniTn5::wssF) were visually similar to WS biofilms, but were substantially thinner and less robust. No obvious differences were observed between WS, WS-6, 9 and 18 biofilm material by fluorescent microscopy.

\section{Effect of cellulose acetylation on physical properties of the $A-L$ biofilm}

Consistent with previous analyses, the only difference between the A-L biofilms produced by WS and the acetylation defective mutant WS-18 (WS mini-Tn5::wSsF) was a reduction of robustness and thickness, rather than a
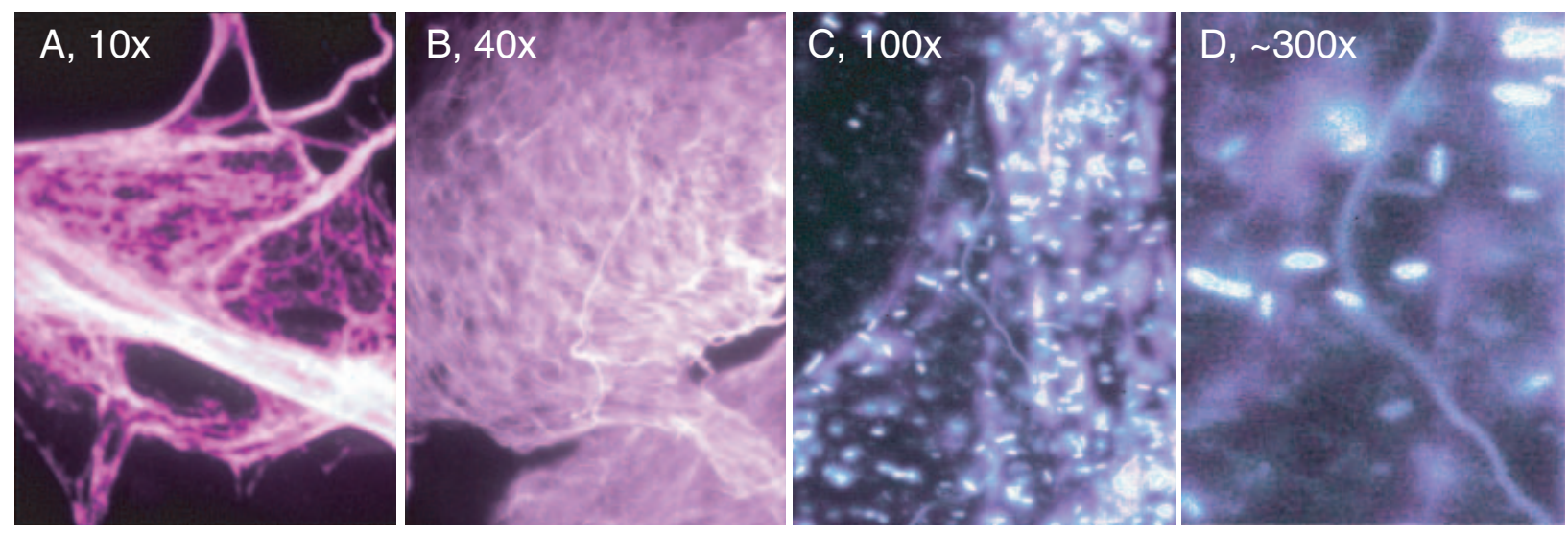

Fig. 5. Microphotographs of WS A-L biofilm samples stained with Calcofluor and viewed at low-high magnification.

A. A large clump of material viewed at low magnification (10x); and B, viewed at medium magnification (40x) showing the variations in structure, including net-like regions, pores and fibres.

C. Bacterial cells associated closely with cellulose fibres seen at high magnification (100x).

D. Enlargement of the central portion of $C$ showing a single cellulose fibre with a width approximately the same as that of a cell $(<0.02 \mu \mathrm{m})$. Note that $P$. fluorescens cells show a high level of autofluorescence. 
change in the amount of cellulose produced or a change in the microscopic structure of the biofilms. Therefore, in order to determine whether differences in cellulose acetylation affected the physical properties of the biofilms per se, we compared the growth rate, biofilm mass and strength of biofilms formed by both WS and WS-18.

First, growth rates of WS and WS-18 were examined in shaking KB cultures to check that there were no significant differences. Both grew rapidly and produced similar maximum growth rates $\left(\Delta O D_{600} / \mathrm{h}\right.$ at mid log phase $\pm \mathrm{SE}, \mathrm{WS}$ : $0.306 \pm 0.009$; WS-18: $0.390 \pm 0.006)$ and final population densities $\left(\mathrm{OD}_{600}\right.$ at $24 \mathrm{~h} \pm \mathrm{SE}$, WS: $1.820 \pm 0.030$; WS-18: $1.937 \pm 0.022)$. Despite these similarities, over a threeday period in a static culture the biomass contained within the WS biofilm increased more rapidly than the biomass contained within the WS-18 biofilm (Fig. 6A).

Within $24 \mathrm{~h}$ of inoculation of a standard KB-containing microcosm, the WS biofilm covered the entire A-L interface. Whereas a similar mass of WS-18 cells grew in the same time period, they did not form a cohesive structure at the A-L interface. The weakness inherent in the WS18 biofilm was clearly demonstrated by the fact that a weight of approximately $0.015 \mathrm{~g}$ was sufficient to cause the biofilm to break. In contrast the WS biofilm was able to support an almost 10-fold greater weight after the same period of growth (Fig. 6B). After $48 \mathrm{~h}$ the weight supported by WS biofilms was approximately 20 -fold greater than that supported by WS-18. Interestingly, the rate of WS biofilm-strengthening decreased between 48 and $72 \mathrm{~h}$, suggesting that once the intact biofilm has formed (i.e. after planar expansion across the A-L interface is complete), the biofilm develops in depth and that expansion in this dimension adds little additional strength to the biofilm. At $72 \mathrm{~h}$ over $75 \%$ of the wet-weight of material in a WS microcosm was retained in the biofilm, and less than $25 \%$ existed in the form of planktonic cells or biofilm debris. In contrast, only $56 \%$ of the WS-18 material was retained in the biofilm and $44 \%$ was planktonic or debris. Although WS-18 is able to expand across the A-L interface to produce a structurally cohesive biofilm, it is less able to retain cells in the biofilm resulting in a slower development of biomass and strength. By plotting the relationship between biofilm wet weight and strength (breaking mass), the substantial differences between WS and WS-18, due to lack of polymer acetylation in the latter, were most apparent (Fig. 6C).

\section{Effect of cellulose acetylation on WS attachment to glass}

Bacterial attachment to a solid surface is the first stage in the development of a biofilm. In A-L biofilms attachment occurs in the meniscus region of the $A-L$ interface. Potentially, poor attachment might result in an underdeveloped or weakened biofilm, and it was possible that a substantially reduced ability to attach to glass might account for the differences between the WS and WS-18 (WS miniTn5::wssF) biofilms. We therefore measured the ability of WS and WS-18 to attach in a glass KB microcosm using a standard Crystal Violet assay (Table 2). Both WS and WS-18 showed greater attachment than the broth colonizing ancestral SM genotype, but no difference was observed between attachment of WS and WS-18, indicating that the weaker WS-18 A-L biofilm is not the result of poor attachment. The WS mini-Tn5::wssB mutant (WS13) also showed relatively high levels of attachment, indicating that attachment to glass was not totally dependent on expression of ACP. Interestingly the WS miniTn5::wspR mutant (WS-4) was much reduced in its ability
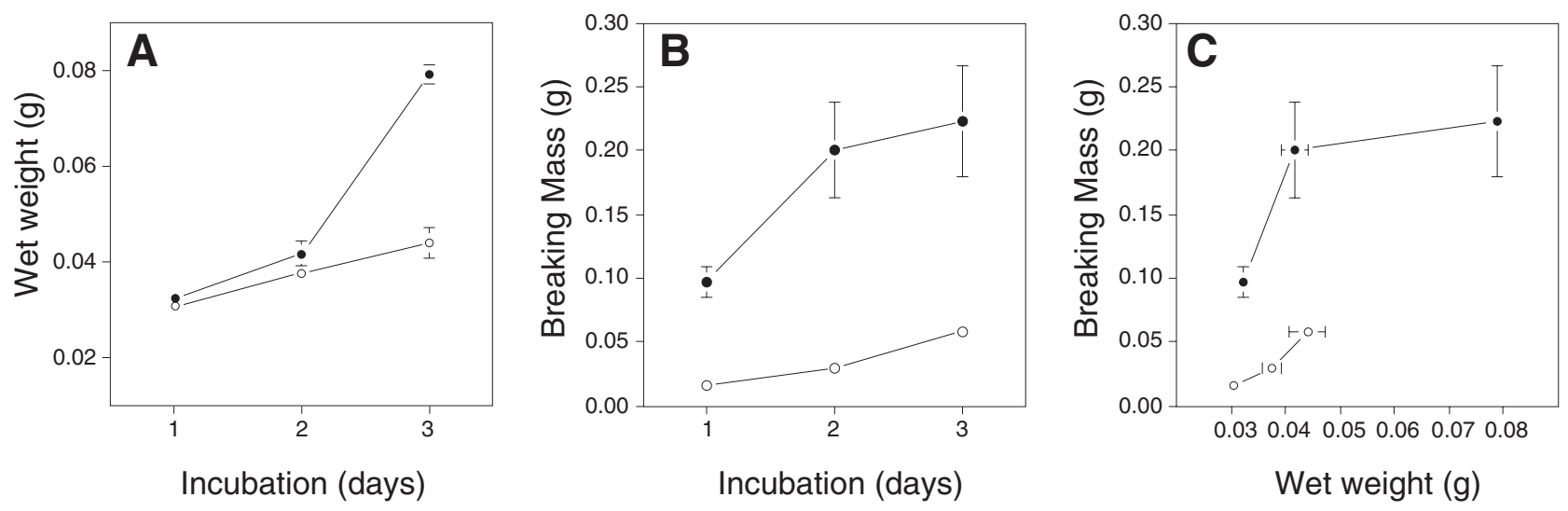

Fig. 6. WS and WS-18 (WS mini-Tn5::wssF) A-L biofilm development differs with respect to total mass and biofilm strength.

A. Biofilm mass (wet weight) of WS (black circles) and WS-18 (white circles).

B. Biofilm strength.

C. Strength/weight ratios. Biofilm masses were calculated from the pooled material of five biofilms with three replicates. Biofilm strengths are shown as the average mass of glass balls needed to break through a biofilm and were determined using five replicates for each day. Means \pm SE are shown. 
Table 2. Bacterial attachment in KB microcosms.

\begin{tabular}{lll}
\hline Strain & Crystal violet staining $\left(\mathrm{OD}_{570}\right)$ & Relative attachment \\
\hline SM & $0.056 \pm 0.020$ & 1.00 \\
WS & $0.187 \pm 0.062$ & 3.34 \\
WS-4 & $0.054 \pm 0.016$ & 0.96 \\
WS-13 & $0.153 \pm 0.032$ & 2.73 \\
WS-18 & $0.178 \pm 0.041$ & 3.18 \\
\hline
\end{tabular}

Strains grown in standard KB (glass) microcosms for $48 \mathrm{~h}$ before analysis. Attachment determined by measuring $\mathrm{OD}_{570}$ of Crystal Violet retained in microcosm vials after washing (mean $\pm \mathrm{SE}, n=8$ ). Relative attachment is calculated relative to SM.

to attach to glass, again indicating that both ACP and at least one other additional component required for surface attachment, probably the non-cellulosic CR-binding component, is dependent upon WspR.

\section{Effect of cellulose acetylation on colony development on agar plates}

The ability of WS to rapidly expand across the A-L interface to form a biofilm has parallels with the rapid colony expansion of WS on agar plates. Just as cellulose acetylation affects biofilm development, it also affects colony development (Fig. 2D and E), with mutants defective in expression of genes responsible for acetylation of cellulose producing normal smooth colonies undistinguishable from SM or WS mutants that are completely defective in wss expression (e.g. WS-13). These differences became most obvious when the ability of the different strains to spread across solid agar surfaces was examined on KB containing differing concentrations of agar (Fig. 7). WS colonies spread maximally at agar concentrations of between 1.3 and $1.7 \%$, producing colonies of more than $15 \mathrm{~mm}$ diameter within 3 days. This pronounced surface spreading was not detected in the acetylation mutant WS mini-Tn5:: wssF (WS-18), in the ACP defective mutant WS
mini-Tn5:: wssB (WS-13), the WspR regulatory mutant WS mini-Tn5::wspR (WS-4), nor in the ancestral broth colonizing genotype (SM).

\section{Discussion}

In water-filled environments colonization of the interface between liquid and gas phases can be selectively advantageous (Rainey and Travisano, 1998). In spatially structured microcosms, spontaneously occurring WS mutants of $P$. fluorescens are strongly favoured by selection on account of their ability to colonize the interface between the nutrient medium and the surrounding air. Ability to colonize this niche stems, to a large part, from the overproduction of a cellulose-like polymer (Spiers et al., 2002). The studies reported here show that this polymer is an acetylated derivative of cellulose. Remarkably, acetylation of the basic cellulose polymer is critical for the formation of the $P$. fluorescens A-L biofilm; genotypes defective in expression of the genes implicated in cellulose acetylation form feeble A-L biofilms and are defective in rapid surface expansion on sold surfaces, even though they continued to produce similar amounts of the core polysaccharide. That such striking phenotypic alterations can be attributed to polymer acetylation reveals how significant the phenotypic effects of subtle modifications to surface expressed polysaccharides can be. In a previous study of biofilms produced by $P$. aeruginosa mutants defective in O-acetylation of alginate (due to mutations in AlgFIJ) a similar marked influence of polymer acetylation on S-L interface biofilm architecture was observed (Nivens et al., 2001).

In order to precisely determine the effects of acetylation on WS A-L biofilm structure we undertook an analysis of physical aspects of the WS biofilm. This revealed the structure to be highly hydrated (over $97 \%$ of the wetweight is water), dependent upon attachment to the glass meniscus for support (i.e. it is not buoyant), and composed
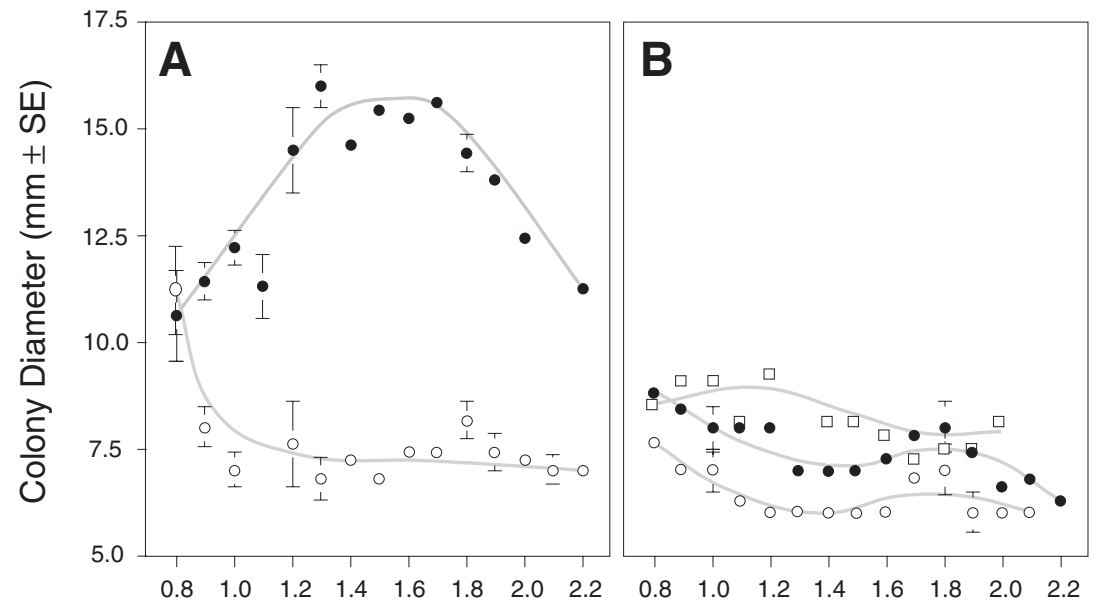

Fig. 7. WS colonies spread more rapidly across agar plates than SM, WS-4 (WS miniTn5:: wspR), WS-13 (WS mini-Tn5::wssB) or WS-18 (WS mini-Tn5::wssF).

A. WS (black circles) and SM (white circles). B. WS-4 (squares), WS-13 (white circles) and WS-18 (black circles). Colonies were inoculated using $5 \mu \mathrm{l}$ drops of culture onto LB plates containing $0.8-2.2 \%(\mathrm{w} / \mathrm{v})$ agar with eight replicates for each strain. Plates were incubated for three days before measurement. Means \pm SE are shown. 
of a vast extra-cellular matrix of ACP fibres that lacked specific organisation or scaled structure, within which bacteria were densely packed. Such structures have been modelled by cellular automaton, and are typical of biofilm systems limited by biomass growth rate rather than by substrate-transport (Picioreanu et al., 1998).

No phenotypic differences were detected among wssFGHIJ mini-Tn5, ISphoA/hah and Cre-deletion mutants, which confirmed the results of a previous study (Spiers et al., 2002) in which transposon insertions in wsSF (WS18), wssH (WS-9) and wssl (WS-6), all of which have polar effects on downstream genes, generated mutants with identical phenotypes (in WS-18, wsSF is disrupted by mini-Tn5 insertion, and wssGHIJ expression prevented by the polar effect of mini-Tn5; cf. in WS-6, wssl is disrupted and only wssJ expression effected). The Cre-

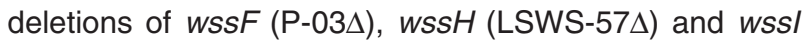
(LSWS-37 $\Delta$ ) mutants show phenotypes indistinguishable from the polar ISphoA/hah and mini-Tn5 mutants of the same genes, indicating that disruption of specific genes, without polar effects on downstream gene expression, was sufficient to produce the SM-phenotype. Our findings here implicate all five genes, wssFGHIJ, in acetylation of cellulose, but only three, wssGHI, are predicted to encode proteins with a known role in polymer acetylation: WssGHI are homologues of AlgFIJ from $P$. aeruginosa (Spiers et al., 2002) which are together necessary and sufficient for $\mathrm{O}$-acetylation of alginate (the three gene products form a single active enzyme; Franklin and Ohman, 1996; Franklin and Ohman, 2002). WssGHI are therefore likely to be functionally equivalent to their alginate acetylating counter parts from $P$. aeruginosa. In this respect it is interesting to note that the $P$. fluorescens SBW25 genome contains, in addition to wssGHI, three clustered genes predicted to encode proteins that show much higher similarity to AlgFIJ from $P$. aeruginosa, than they do to WssGHI ( 70\% identity compared with $\sim 30 \%$ identity to WssGHI). This indicates that $P$. fluorescens SBW25 contains two sets of genes for polymer acetylation - one involved in cellulose acetylation and the other in acetylation of alginate (a polymer that $P$. fluorescens SBW25 is known to produce). Whether or not the two acetylation complexes show substrate specificity remains to be determined (the inability of the putative AlgFIJ homologues to rescue wssGHI mutants may simply reflect lack of algFIJ expression under the conditions used here), but given the significant amino acid sequence differences among the two complexes, this is a reasonable possibility.

Homologues of WssF are present in just three cellulose biosynthetic gene clusters from a total of 19 bacteria currently known, or predicted on the basis of in silico analysis, to make cellulose. This alone suggests that WssF is not generally required for synthesis of the basic cellulose polymer. Intriguingly, in the three genomes where WssF is present ( $P$. fluorescens SBW25, $P$. syringae pathovar tomato DC3000 and G. xylinus JCM 7664) it is linked to genes predicted to be involved in cellulose acetylation. In $P$. syringae pathovar tomato DC3000 the cellulose biosynthetic gene cluster harbours not only a homologue of WssF (BcsX) (Römling, 2002), but the adjacent downstream genes are also homologues of WssGHI (53\%, $81 \%$ and $74 \%$ amino acid identity, respectively, to their $P$. fluorescens SBW25 counterparts), which strongly suggests that this bacterium produces ACP. In G. xylinus strain JCM 7664 the WssF homologue, bcsX, is found between bcsB (cellulose synthase) and bcsY. BcsY is predicted to encode a transacylase, which led Umeda et al. (1999) to propose that JCM 7664 produced an acetylated form of cellulose, although this has not been experimentally verified. Whereas the function of WssF remains to be determined, its sole occurrence in the genomes of bacteria predicted to make acetylated cellulose suggests that it plays a role in acetylation. This prediction is further strengthened by the fact that WssF shows features compatible (albeit weakly) with a newly established Pfam family of GDSL-like lipases and acylhydrolases $(E$-value $=0.31)$ (Bateman et al., 2000). Given that BcsX from G. xylinus and WssF from $P$. syringae pathovar tomato DC3000 also fall within this Pfam family, we postulate that WssF/BcsX is involved in the presentation of acyl groups to enzymes (WssGHI) directly involved in the acetylation of cellulose.

The role of the terminal gene of the wss operon, wssJ, is also unknown, but like wssFGHI, it is not required for production of the core cellulose polymer. WssJ, like WssA, shows similarity to the cell cycle protein MinD from a range of bacteria (Spiers et al., 2002), but unlike WssA, which is encoded by the first gene of many cellulose biosynthetic operons (Römling, 2002), homologues of WssJ are not present in the other cellulose biosynthetic gene clusters described to date - not even in $P$. syringae pathovar tomato DC3000, which uniquely with $P$. fluorescens SBW25, encodes homologues of WssFGHI (in addition to homologues of WssABCDE). Previously we had proposed that WssA and WssJ are required for localizing the cellulose synthase complex to cell poles. It is possible that WssA specifically acts to localize the core cellulose synthase complex (WssBCDE), whereas WssJ localizes the enzymes involved in acetylation (WssFGHI). The finding that LSWS ISphoA/hah::wssI (LSWS-57) (in which wssl is disrupted by ISphoA/hah insertion, and the expression of wssJ prevented by the polar effect of the transposon) and the Cre-deletion LSWS-57D (in which only wss/ function is destroyed) suggests that either the functional role of WssJ is inseparable from that of the WssFGHI acetylation complex, or that WssJ is not essential for WssFGHI acetylation. These hypotheses are cur- 
rently being tested using both Wss-GFP translational fusions and targeted gene knockouts.

Analysis of the extracellular matrix associated with rdar morphs of $S$. typhimurium showed that it is composed of both cellulose and thin aggregative fimbriae (Römling, 2002; Zogaj et al., 2001). Our finding that the ACPdefective WS-13 mutant (WS mini-Tn5::wssB) overproduced a Congo Red-binding component that could not be eliminated by cellulase treatment, strongly suggested the existence of an additional non-cellulosic component in the WS extracellular matrix. This was subsequently confirmed by showing that WS-13 produced no Calcofluor staining material (as assessed by fluorescent microscopy), but did show evidence of a fimbrial-like component when examined by TEM, which was also evident in WS, but not present in the ancestral SM genotype. Moreover, the results of the attachment assays demonstrated that the non-cellulosic CR-binding component is largely responsible for attachment to glass surfaces, and that ACP appeared to play little role in cell-glass attachment. The genetic identity of the fimbrial-like component is currently being sort.

There is growing awareness of the role that GGDEF regulators (Pei and Grishin, 2001) play in modulating expression of cell surface components and preparing cells for cell-cell and cell-surface interaction (Aldridge et al., 2003). For example, GGDEF regulators have been implicated in the modulation of surface structures in Caulobacter crescentus (Aldridge and Jenal, 1999; Aldridge et al., 2003), G. xylinus (Tal et al., 1998), Rhizobium leguminosarum (Ausmees et al., 1999), S. typhimurium (Römling and Rohde, 1999; Zogaj et al., 2001), Yersinia pestis (Jones et al., 1999), and in both P. aeruginosa (D'Argenio et al., 2002; Drenkard and Ausubel, 2002) and P. fluorescens (Spiers et al., 2002). In P. aeruginosa the formation of wrinkled colonies and concomitant ability to colonize the A-L interface involves at least two loci whose expression is dependent upon the GGDEF regulator WspR. These loci include the cup genes which are required for expression of fimbriae, and several genes implicated in glycogen synthesis (Vallet et al., 2001; D'Argenio et al., 2002). On the basis of the results obtained here, it appears that WspR from $P$. fluorescens, which is functionally interchangeable with WspR from $P$. aeruginosa (D'Argenio et al., 2002), controls expression of both ACP and the putative fimbrial component detected by the Congo Red assays and by TEM. Given that ACP is implicated in cell-cell contact and the putative fimbrial component in cell-surface contact, their combined control by WspR lends further support to the view that GGDEF regulators play a role in the co-ordination of surface contact and colonization strategies across a range of bacteria (Aldridge et al., 2003).

In addition to dramatic effects on the physical properties of the A-L biofilm that arise from defects in acetylation of cellulose, analysis of the surface spreading behaviour of WS and ACP-defective mutants led us to reconsider the possible ecological significance of ACP production. Under normal environmental conditions, wild-type (i.e. SM) SBW25 probably expresses ACP as a result of an as yet unidentified external stimulus detected by the Wsp chemosensory system via the $\mathrm{WspR}$ response regulator. It is possible that small amounts of ACP act as a cohesive factor, which, in concert with the fimbrial-like component, serve to control surface colonization.

\section{Experimental procedures}

\section{Strains and growth conditions}

The Pseudomonas fluorescens strains used in this work are derivatives of $P$. fluorescens SBW25 (Table 3). Bacteria were grown in either KB or LB media (King et al., 1954; Miller, 1972 ) at $28^{\circ} \mathrm{C}$. Congo Red (SIGMA) was added to LB (no $\mathrm{NaCl})$ plates at a final concentration of $0.001 \%(\mathrm{w} / \mathrm{v})$. Chloramphenicol was used at $20 \mu \mathrm{g} \mathrm{ml}^{-1}$, kanamycin at $75 \mu \mathrm{g} \mathrm{ml}^{-1}$ and tetracycline at $25 \mu \mathrm{g} \mathrm{ml}^{-1}$. Biofilms were produced in $25 \mathrm{ml}$ glass vials containing $6 \mathrm{ml} \mathrm{KB}$ inoculated with an overnight culture and incubated for $3-7$ days at $28^{\circ} \mathrm{C}$ (after Rainey and Travisano, 1998).

\section{Production of ISphoA/hahs mutants}

Cre-deletions of LSWS ISphoA/hah mutants were generated according to the method of Bailey and Manoil (2002), except that $P$. fluorescens strains were incubated at $45^{\circ} \mathrm{C}$ for $15 \mathrm{~min}$ before conjugation, and chloramphenicol at $20 \mu \mathrm{g} \mathrm{ml}^{-1}$ was used to counter-select against E. coli. Appropriate Credeletants were identified as tetracycline-sensitive colonies by patch-plating onto LB plates with and without $25 \mu \mathrm{g} \mathrm{ml}^{-1}$ tetracycline.

\section{Microscopy}

Biofilm material taken directly from microcosms or colonies was stained with $10 \mu \mathrm{M}$ Calcofluor (SIGMA Fluorescent Whitener 28) in LB or KB for 30-60 min before fluorescent microscopy. Cells were fixed in gluteraldehyde and stained with osmonium tetroxide/potassium ferocyanide/uranyl acetate before embedding in resin and thin-sectioning. These sections were placed on EM grids and stained further with lead citrate before transmission electron microscopy. Fluorescent and TEM images were manipulated in Adobe Photoshop using only the 'auto-level' and 'auto-contrast' functions.

\section{Attachment assay}

Bacterial attachment was determined quantitatively using Crystal Violet staining after O'Toole et al. (1999). Microcosm vials were emptied and washed vigorously with deionised water. One $\mathrm{ml}$ 0.05\% (w/v) Crystal Violet (SIGMA) was added and mixed for two minutes. The vials were then emptied and washed with deionised water. The stain was eluted with $5 \mathrm{ml}$ 
Table 3. P. fluorescens SBW25 strains used in this study.

\begin{tabular}{|c|c|c|}
\hline Strain & Genotype/phenotype & Ref/source \\
\hline SM & Wild-type SBW25 which does not form biofilm or express cellulose. & Rainey and Bailey (1996) \\
\hline WS & Biofilm-forming and cellulose-expressing strain evolved from SM. & Spiers et al. (2002) \\
\hline WS-4 & WS mini-Tn5::wspR. Does not form biofilm or express cellulose. & Spiers et al. (2002) \\
\hline WS-6 & WS mini-Tn5:: wssl. ${ }^{\text {a }}$ Forms weak biofilm and expresses cellulose. & Spiers et al. (2002) \\
\hline WS-9 & WS mini-Tn5::wssH. Forms weak biofilm and expresses cellulose. & Spiers et al. (2002) \\
\hline WS-13 & WS mini-Tn5:: wssB. Does not form biofilm or express cellulose. & Spiers et al. (2002) \\
\hline WS-18 & WS mini-Tn5::wssF. Forms weak biofilm and expresses cellulose. & Spiers et al. (2002) \\
\hline LSWS-37 & WS ISphoA/hah:: wssI. Forms weak biofilm and expresses cellulose. & S. Gehrig \\
\hline LSWS-37ム & Cre-mediated deletion of LSWS-37. Forms weak biofilm and expresses cellulose. & This work \\
\hline LSWS-48 & WS ISphoA/hah::wssB. Does not form biofilm or express cellulose. & S. Gehrig \\
\hline LSWS-48 $\Delta$ & Cre-mediated deletion of LSWS-48. Does not form biofilm or express cellulose. & This work \\
\hline LSWS-57 & WS ISphoA/hah:: wssH. Forms weak biofilm and expresses cellulose. & S. Gehrig \\
\hline LSWS-57 & Cre-mediated deletion of LSWS-57. Forms weak biofilm and expresses cellulose. & This work \\
\hline $\mathrm{P}-03$ & WS ISphoA/hah::wssF. Forms weak biofilm and expresses cellulose. & S. Gehrig \\
\hline $\mathrm{P}-03 \Delta$ & Cre-mediated deletion of P-03. Forms weak biofilm and expresses cellulose. & This work \\
\hline
\end{tabular}

a. The position of the mini-Tn5 transposon in WS-6 was incorrectly presented in Spiers et al. (2002). The position in wss/ has been re-confirmed by sequence analysis

ethanol with vigorous shaking for $30 \mathrm{~min}$ and the $\mathrm{OD}_{570}$ determined.

\section{Congo Red-binding assay}

Cellulose production in colonies was determined quantitatively using Congo Red (CR). Colonies were resuspended in $\mathrm{KB}$ and cell density determined by measuring the $\mathrm{OD}_{600}$. The cells were resuspended in $1 \mathrm{ml} 0.005 \%$ (w/v) CR (SIGMA) and incubated for $2 \mathrm{~h}$ at $37^{\circ} \mathrm{C}$. Cells were then removed by centrifugation. The amount of $\mathrm{CR}$ remaining in the supernatant was determined by measuring the $\mathrm{OD}_{490}$ of the solution and comparison with appropriate $\mathrm{CR}$ standards to obtain $\mathrm{mg}$ CR. CR-binding was expressed as $\mathrm{mg} \mathrm{CR} / \mathrm{OD}_{600}$. For binding assays using cellulase treatment, cells were resuspended in $5 \mathrm{mM}$ MES (pH 5.0), $170 \mathrm{mM} \mathrm{NaCl}$, and digested with $50 \mathrm{U}$ Aspergillus niger cellulase (ICN Biomedical) for $1 \mathrm{~h}$ at $37^{\circ} \mathrm{C}$ before incubation with $\mathrm{CR}$.

\section{Isolation of biofilm carbohydrate material}

Biofilms were harvested from 480 microcosms by centrifugation at $10000 \mathrm{~g}$ for $5 \mathrm{~min}$, re-suspended in $200 \mathrm{ml} 1 \mathrm{M} \mathrm{NaCl}$, 100 mM EDTA, 20 mM Tris-HCl (pH 8.0), 0.5\% (w/v) Sarkosyl. The biofilms were extensively digested with $1 \mathrm{mg} \mathrm{ml}^{-1}$ lysozyme (SIGMA) and then $1.5 \mathrm{mg} \mathrm{ml}^{-1}$ Proteinase $\mathrm{K}$ (SIGMA) over a period of $96 \mathrm{~h}$ at $37^{\circ} \mathrm{C}$. The digested material was extensively washed with $100 \mathrm{mM} \mathrm{CaCl}_{2}$ before a final wash in deionized water and freeze-drying.

\section{Carbohydrate analyses}

Composition and linkage analysis used freeze-dried samples prepared as above. For compositional analysis, the samples were hydrolysed using freshly prepared $1 \mathrm{M}$ methanolic- $\mathrm{HCl}$ for $16 \mathrm{~h}$ at $80^{\circ} \mathrm{C}$. The released sugars were derivatized with Tr-Sil and the sample was analysed by GC-MS using a Sp2330 Supelco column. For linkage analysis, the sample was first methylated after Ciucanu and Kerek (1984), then hydrolysed in $2 \mathrm{M}$ TFA at $121^{\circ} \mathrm{C}$ for $2 \mathrm{~h}$. The hydrolysed carbohydrate was reduced with sodium borodeuteride at room temperature then acetylated using acetic anhydride at $120^{\circ} \mathrm{C}$ for $3 \mathrm{~h}$. The derivatized sample was then analysed by GC-MS. In both analyses, myo-inositol was added to the sample as an internal standard.

For NMR analysis, the freeze-dried samples were deuterium-exchanged by repeated evaporation from $\mathrm{CD}_{3} \mathrm{OD}$ and dissolved in $0.5 \mathrm{ml}$ of $\mathrm{CD}_{3} \mathrm{OD}$. A $1-\mathrm{D}$ proton spectrum was acquired on a Varian Inova $500 \mathrm{MHz}$ spectrometer at $308^{\circ} \mathrm{K}$ $\left(35^{\circ} \mathrm{C}\right)$. Proton chemical shifts were measured relative to internal TMS standard ( $\delta=0.000$ p.p.m.).

\section{Acknowledgements}

This work was supported in part by the BBSRC and a University Challenge Seed Fund to A.J.S. and P.B.R. J.B. was supported by a Christopher Welch Fellowship. We acknowledge the kind help of Harry Flint and David Harvey in the initial chemical analysis of ACP. The Complex Carbohydrate Research Centre (University of Georgia, USA) were contracted to perform further carbohydrate analyses for this work. Preliminary sequence data used for BLAST analysis was obtained from the P. fluorescens SBW25 Sequencing Group at the Sanger Institute (http://www.sanger.ac.uk) and The Institute for Genomic Research (TIGR) (http://www.tigr.org). We thank John Baker for his help with photography and Julie Stansfield for her technical support. We also acknowledge the help of the Referee's comments in the preparation of this manuscript.

\section{References}

Aldridge, P., and Jenal, U. (1999) Cell cycle-dependent degradation of a flagellar motor component requires a noveltype response regulator. Mol Microbiol 32: 379-391.

Aldridge, P., Paul, R., Goymer, P.J., Rainey, P.B., and Jenal, U. (2003) Role of the GGDEF regulator PleD in polar 
development of Caulobacter crescentus. Mol Microbiol 47: 1695-1708.

Ausmees, N., Jonsson, H., Hoglund, S., Ljunggren, H., and Lindberg, M. (1999) Structural and putative regulatory genes involved in cellulose synthesis in Rhizobium leguminosarum bv. trifolii. Microbiol 145: 1253-1262.

Bailey, J., and Manoil, C. (2002) Genome-wide internal tagging of bacterial exported proteins. Nat Biotechnol 20: 839-842.

Bateman, A., Birney, E., Durbin, R., Eddy, S.R., Howe, K.L., and Sonnhammer, E.L. (2000) The Pfam Protein Families Database. Nucleic Acid Res 28: 263-266.

Ciucanu, I., and Kerek, F. (1984) A simple and rapid method for the permethylation of carbohydrates. Carbohydr Res 131: 209-217.

Collinson, S.K., Doig, P.C., Doran, J.L., Clouthier, S., Trust, T.J., and Kay, W.W. (1993) Thin, aggregative fimbriae mediate binding of Salmonella enteritidis to fibronectin. $J$ Bacteriol 175: 12-18.

Costerton, J.W., Lewandowski, Z., Cladwell, D.E., Korber, D.R., and Lappin-Scott, H.M. (1995) Microbial biofilms. Annu Rev Microbiol 49: 711-745.

D'Argenio, D.A., Calfee, M.W., Rainey, P.B., and Pesci, E.C. (2002) Autolysis and autoaggregation in Pseudomonas aeruginosa colony morphology mutants. J Bacteriol 184: 6481-6489.

Davey, M.E., and O'Toole, G.A. (2000) Microbial biofilms: from ecology to molecular genetics. Microbiol Mol Biol Rev 64: 847-867.

Drenkard, E., and Ausubel, F.M. (2002) Pseudomonas biofilm formation and antibiotic resistance are linked to phenotypic variation. Nature 416: 740-743.

Franklin, M.J., and Ohman, D.E. (1996) Identification of algl and algJ in the Pseudomonas aeruginosa alginate biosynthetic gene cluster which are required for alginate $\mathrm{O}$ acetylation. J Bacteriol 178: 2186-2195.

Franklin, M.J., and Ohman, D.E. (2002) Mutant analysis and cellular localization of the Algl, AlgJ, and AlgF proteins required for $\mathrm{O}$ acetylation of alginate in Pseudomonas aeruginosa. J Bacteriol 184: 3000-3007.

Hamilton, W.D., and May, R.M. (1977) Dispersal in stable habitats. Nature 269: 578-581.

Hammar, M., Arnqvist, A., Bian, Z., Olsen, A., and Normark, S. (1995) Expression of two csg operons is required for production of fibronectin- and congo red-binding curli polymers in Escherichia coli K-12. Mol Microbiol 18: 661-670.

Jones, H.A., Lillard, J.W., Jr, and Perry, R.D. (1999) HmsT, a protein essential for expression of the haemin storage (Hms+) phenotype of Yersinia pestis. Microbiology 145: 2117-2128.

King, E.O., Ward, M.K., and Raney, D.C. (1954) Two simple media for the demonstration of pyocyanin and fluorescin. $J$ Laboratory Clin Med 44: 301-307.

Knirel, Y.A., Helbig, J.H., and Zahringer, U. (1996) Structure of a decasaccharide isolated by mild acid degradation and dephosphorylation of the lipopolysaccharide of Pseudomonas fluorescens strain ATCC 49271. Carbohydr Res 283: 129-139.

Kuchma, S.L., and O'Toole, G.A. (2000) Surface-induced and biofilm-induced changes in gene expression. Curr Opin Biotechnol 11: 429-433.
Lappin-Scott, H.M., and Bass, C. (2001) Biofilm formation: attachment, growth and detachment of microbes from surfaces. Am J Infect Control 29: 250-261.

Lawrence, J.R., Korber, D.R., Hoyle, B.D., Costerton, J.W., and Cadwell, D.E. (1991) Optical sectioning of microbial biofilms. J Bacteriol 173: 6558-6567.

Miller, J. (1972) Experiments in Molecular Genetics. Cold Spring Harbor, NY: Cold Spring Harbor Laboratory Press.

Naberezhnykh, G.A., Khomenko, V.A., Isakov, V.V., Elkin, I.N., and Soloveva, T.F. (1987) In Russian: [3-(3-hydroxy2,3-dimethyl-5-oxoprolyl) amino-3,6-dideoxy-D-glucose: a new amino sugar of the antigenic polysaccharide from Pseudomonas fluorescens]. Bioorg Khim 13: 1428-1429.

Nivens, D.E., Ohman, D.E., Williams, J., and Franklin, M.J. (2001) Role of alginate and its $O$ acetylation in formation of Pseudomonas aeruginosa microcolonies and biofilms. $J$ Bacteriol 183: 1047-1057.

O'Toole, G.A., Pratt, L.A., Watnick, P.I., Newman, D.K., Weaver, V.B., and Kolter, R. (1999) Genetic approaches to study of biofilms. Methods Enzymol 310: 91-109.

Pei, J., and Grishin, N.V. (2001) GGDEF domain is homologous to adenylyl cyclase. Proteins 42: 210-216.

Picioreanu, C., van Loosdrecht, M.C., and Heijnen, J.J. (1998) Mathematical modelling of biofilm structure with a hybrid differential-discrete cellular automaton approach. Biotechnol Bioeng 58: 101-116.

Rainey, P.B., and Bailey, M.J. (1996) Physical map of the Pseudomonas fluorescens SBW25 chromosome. Mol Microbiol 19: 521-533.

Rainey, P.B., and Travisano, M. (1998) Adaptive radiation in a heterogeneous environment. Nature 394: 69-72.

Roberts, I.S. (1996) The biochemistry and genetics of capsular polysaccharide production in bacteria. Annu Rev Microbiol 50: 285-315.

Rocchetta, H.L., Burrows, L.L., and Lam, J.S. (1999) Genetics of O-antigen biosynthesis in Pseudomonas aeruginosa. Microbiol Mol Biol Rev 63: 523-553.

Römling, U. (2002) Molecular biology of cellulose production in bacteria. Res Microbiol 153: 205-212.

Römling, U., and Rohde, M. (1999) Flagella modulate the multicellular behaviour of Salmonella typhimurium on the community level. FEMS Microbiol Lett 180: 91-102.

Shashkov, A.S., Paramonov, N.A., Veremeychenko, S.P., Grosskurth, H., Zdorovenko, G.M., Knirel, Y.A., and Kochetkov, N.K. (1998) Somatic antigens of pseudomonads: structure of the O-specific polysaccharide of Pseudomonas fluorescens biovar B, strain IMV 247. Carbohydr Res 306: 297-303.

Solano, C., Garcia, B., Valle, J., Berasain, C., Ghigo, J.-M., Gamazo, C., and Lasa, I. (2002) Genetic analysis of Salmonella enteritidis biofilm formation: critical role of cellulose. Mol Microbiol 43: 793-808.

Spiers, A.J., Kahn, S.G., Travisano, M., Bohannon, J., and Rainey, P.B. (2002) Phenotypic Evolution in Pseudomonas fluorescens. 1. Determinants of Wrinkly Spreader fitness and the cause of an evolutionary transition. Genetics 161: 33-46.

Stephens, C. (2002) Microbiology: breaking down biofilms. Curr Biol 12: 132-134.

Sullivan, S.M., and Maddock, J.R. (2000) Bacterial division: Finding the dividing line. Current Biol 10: R249-R252. 
Sutherland, I.W. (2001) The biofilm matrix - an immobilized but dynamic microbial environment. Trends Microbiol 9: 222-227.

Tal, R., Wong, H.C., Calhoon, R., Gelfand, D., Fear, A.L., Volman, G., et al. (1998) Three cdg operons control cellular turnover of cyclic di-GMP in Acetobacter xylinum: genetic organization and occurrence of conserved domains in isoenzymes. J Bacteriol 180: 4416-4425.

Umeda, Y., Hirano, A., Ishibashi, M., Akiyama, H., Onizuka, T., Ikeuchi, M., and Inoue, Y. (1999) Cloning of cellulose synthase genes from Acetobacter xylinum JCM 7664: implication of a novel set of cellulose synthase genes. DNA Res 30: 109-115.

Vallet, I., Olson, J.W., Lory, S., Lazdunski, A., and and Filloux, A. (2001) The chaperone/usher pathways of Pseudomonas aeruginosa: identification of fimbrial gene clusters (cup) and their involvement in biofilm formation. Proc Natl Acad Sci USA 98: 6911-6916.

Veremeichenko, S.N. (1998) Characteristics of Pseudomonas fluorescens IMV 1433 (Biovar I) lipopolysaccharide. Mikrobiologiia 67: 505-511 (in Russian).

Veremeichenko, S.N., and Zdorovenko, G.M. (1994) Characteristics of Pseudomonas fluorescens lipoplysaccharide. Mikrobiologiia 63: 831-839 (in Russian).

Veremeichenko, S.N., and Zdorovenko, G.M. (1996) Characterization of the lipopolysaccharide from the Pseudomonas fluorescens strain IMV 472 (biovar I). Mikrobiologiia 65: 318-325 (in Russian).

Watnick, P.I., and Kolter, R. (1999) Steps in the development of a Vibrio cholerae El Tor biofilm. Mol Microbiol 34: 586595.

Watnick, P., and Kolter, R. (2000) Biofilm, City of microbes. J Bacteriol 182: 2675-2679.

Weiner, R., Seagren, E., Arnosti, C., and Quintero, E. (1999) Bacterial survival in biofilms: probes for exopolysaccharide and its hydrolysis, and measurements of intra- and interphase mass fluxes. Methods Enzymol 310: 403-426.

Wilson, W. (2001) Bacterial biofilms and human disease. Sci Prog 84: 235-254.

Wimpenny, J., Manz, W., and Szewzyk, U. (2000) Heterogeneity in biofilms. FEMS Microbiol Rev 24: 661671.

Zdorovenko, G.M., Gvozdiak, R.I., Gubanova, N.I., Afonina, G.B., and Zdorovenko, E.L. (1999) Characteristics of lipopolysaccharide from Pseudomonas fluorescens (biovar I). Mikrobiologiia 68: 330-339 (in Russian).

Zogaj, X., Nimtz, M., Rohde, M., Bokranz, W., and Römling, U. (2001) The multicellular morphotypes of Salmonella typhimurium and Escherichia coli produce cellulose as the second component of the extracellular matrix. Mol Microbiol 39: 1452-1463. 